OPEN ACCESS

Edited by:

Kotambail Venkatesha Udupa, University College Dublin, Ireland

Reviewed by:

Sergey V. Ryzhov

Maine Medical Center, USA

Yi Zhu,

Tianjin Medical University, China

*Correspondence:

Jeannette Kunz Jeanette.kunz@nu.edu.kz

Specialty section:

This article was submitted to Inflammation Pharmacology,

a section of the journal

Frontiers in Pharmacology

Received: 01 September 2016 Accepted: 16 November 2016 Published: 01 December 2016

Citation:

Zhubanchaliyev A, Temirbekuly A, Kongrtay K, Wanshura LC and Kunz J

(2016) Targeting

Mechanotransduction at the Transcriptional Level: YAP and BRD4 Are Novel Therapeutic Targets for the

Reversal of Liver Fibrosis.

Front. Pharmacol. 7:462.

doi: 10.3389/fphar.2016.00462

\section{Targeting Mechanotransduction at the Transcriptional Level: YAP and BRD4 Are Novel Therapeutic Targets for the Reversal of Liver Fibrosis}

\author{
Altynbek Zhubanchaliyev ${ }^{1,2}$, Aibar Temirbekuly ${ }^{1}$, Kuralay Kongrtay ${ }^{1}$, Leah C. Wanshura ${ }^{3}$ \\ and Jeannette Kunz ${ }^{1 *}$ \\ ${ }^{1}$ Department of Biology, School of Science and Technology, Nazarbayev University, Astana, Kazakhstan, ${ }^{2}$ Department of \\ Biotechnology and Microbiology, Faculty of Natural Sciences, L.N.Gumilyov Eurasian National University, Astana, \\ Kazakhstan, ${ }^{3}$ BioScribia, Lafayette, CO, USA
}

Liver fibrosis is the result of a deregulated wound healing process characterized by the excessive deposition of extracellular matrix. Hepatic stellate cells (HSCs), which are activated in response to liver injury, are the major source of extracellular matrix and drive the wound healing process. However, chronic liver damage leads to perpetual HSC activation, progressive formation of pathological scar tissue and ultimately, cirrhosis and organ failure. HSC activation is triggered largely in response to mechanosignaling from the microenvironment, which induces a profibrotic nuclear transcription program that promotes HSC proliferation and extracellular matrix secretion thereby setting up a positive feedback loop leading to matrix stiffening and self-sustained, pathological, HSC activation. Despite the significant progress in our understanding of liver fibrosis, the molecular mechanisms through which the extracellular matrix promotes HSC activation are not well understood and no effective therapies have been approved to date that can target this early, reversible, stage in liver fibrosis. Several new lines of investigation now provide important insight into this area of study and identify two nuclear targets whose inhibition has the potential of reversing liver fibrosis by interfering with HSC activation: Yes-associated protein (YAP), a transcriptional co-activator and effector of the mechanosensitive Hippo pathway, and bromodomain-containing protein 4 (BRD4), an epigenetic regulator of gene expression. YAP and BRD4 activity is induced in response to mechanical stimulation of HSCs and each protein independently controls waves of early gene expression necessary for HSC activation. Significantly, inhibition of either protein can revert the chronic activation of HSCs and impede pathological progression of liver fibrosis in clinically relevant model systems. In this review we will discuss the roles of these nuclear co-activators in HSC activation, their mechanism of action in the fibrotic process in the liver and other organs, and the potential of targeting their activity with small molecule drugs for fibrosis reversal.

Keywords: fibrosis, myofibroblasts, mechanotransduction, Hippo pathway, YAP, bromodomain, BRD4 


\section{INTRODUCTION}

Chronic fibroproliferative disease is a major cause of death in the industrialized world, placing a substantial burden on healthcare systems (Mehal et al., 2011; Rockey et al., 2015). In the liver, chronic injury from a variety of etiologies, including hepatitis, obesity/metabolic syndrome, and inflammation, leads to liver fibrosis (Ballestri et al., 2016). While fibrosis due to hepatitis can be treated with antiviral therapies, nonviral sources remain difficult to treat (Poordad et al., 2014; Zeuzem et al., 2014) and once progressed, treatment is limited to slowing escalation of the disease, but cannot reverse liver fibrosis (Friedman et al., 2013). The only treatment option for end-stage liver disease to date is liver transplantation; but this is limited by availability of donor organs and associated with significant longterm complications and costs. While the late stages of liver fibrosis are irreversible, mounting evidence from relevant rodent models and some patient studies suggest that the early stages of fibrosis and, potentially, even cirrhosis are reversible (Liu et al., 2013). Thus, there is an urgent need for the development of more effective therapeutic strategies that aim to target liver fibrosis and better knowledge of the molecular events underlying the early stages of fibrosis holds the key to blocking disease progression.

Regardless of tissue type and etiology, fibrosis is the result of an aberrant wound healing response to tissue injury or inflammation, characterized by excessive remodeling of the extracellular matrix and progressive scarring (Mehal et al., 2011; Rockey et al., 2015). This wound healing response is largely driven by mechanotransduction-that is, intracellular signaling that is initiated by and mediated through mechanical forces generated by physical interactions between cells and the extracellular microenvironment. During the chronic fibrotic response, mechanical signaling initiated by extracellular matrix proteins, such as interstitial collagens, leads to a positive feedback loop by which fibrotic cells produce an excess of extracellular matrix proteins. This, in turn, leads to increased tissue stiffness and further activation of fibrotic cells. In the liver, this process is mainly mediated by HSCs that become activated and differentiate into myofibroblasts that are proliferative and fibrogenic (Iwaisako et al., 2014). The switch from quiescent HSC to myofibroblast involves mechanotransduction-induced gene transcription of fibrillary collagens and alpha smooth muscle actin ( $\alpha$-SMA), leading to stress fiber formation, extracellular matrix deposition and increased cell-extracellular matrix contact. Ultimately, the resulting tissue contraction promotes chronic HSC activation through a positive feedback loop involving the microenvironment and HSCs that perpetuates HSC activation (Hinz et al., 2007; Friedman, 2008; Butcher et al., 2009; Mederacke et al., 2013). Importantly, while fibrogenic signaling is activated during the earliest disease stages, extracellular matrix accumulation leading to fibrotic scar development only occurs during the course of chronic tissue injury. Uncovering the molecular mechanisms that drive early steps in HSC activation and sustain the deposition of extracellular matrix therefore is key to halting the mechanosensitive feedback loop and reversing the fibrotic response.
Recent studies suggest that the gene transcription program necessary for HSC activation is controlled, at least in part, by two transcriptional regulators: Yes-associated protein (YAP) and bromodomain-containing protein 4 (BRD4) (Ding et al., 2015; Mannaerts et al., 2015). Although these transcriptional regulators appear to function independently of each other through unique molecular mechanisms during stellate cell activation, each is regulated by the microenvironment during liver fibrosis. Importantly, pharmacologic inhibition of YAP or BRD4 with small molecules that disrupt their respective interactions with cofactors has produced promising antifibrotic responses in murine liver fibrosis models (Ding et al., 2015; Mannaerts et al., 2015) and thus, represents an attractive therapeutic approach to treat liver fibrosis in patients. In this review, we will discuss the roles of these transcription factors in fibroproliferative diseases, their potential contribution to mechanotransduction during HSC activation, and the potential of targeting these proteins that mediate extracellular matrix stiffness-induced gene expression to treat patients with fibrosis.

\section{HEPATOCELLULAR STELLATE CELLS MEDIATE LIVER FIBROSIS}

Liver fibrosis is mediated by myofibroblasts that in mice arise from two cell types: HSCs are the major source of myofibroblasts in hepatocellular injury (carbon tetrachloride; $\mathrm{CCl}_{4}$ ) induced fibrosis, whereas portal fibroblasts are the major source of myofibroblasts in response to cholestatic injury (bile duct ligation) induced fibrosis (Iwaisako et al., 2014). Interestingly, HSCs become activated by portal fibroblasts with longer durations (14-20 days) of bile duct ligation-induced cholestatic liver injury, thereafter promoting chronic fibrosis. These cholestatic injury-induced HSCs are "portal fibroblast-like," possessing a gene expression profile-including Thyl and elastin-similar to portal fibroblast-derived myofibroblasts and distinct from that of hepatocellular injury-induced myofibroblasts, which are characterized by Vitamin A and glial fibrillary acidic protein (GFAP) expression (Mederacke et al., 2013; Iwaisako et al., 2014). Thus, HSC activation ultimately represents the major source of chronic liver fibrosis in both hepatocellular and cholestatic models.

In healthy livers, quiescent, vitamin A-rich HSCs reside in the space of Disse (Iwaisako et al., 2014). In response to liver injury (Figure 1), HSCs proliferate and deposit extracellular matrix components such as interstitial collagens and matrix metalloproteinases (MMPs) (Friedman, 2008; Mederacke et al., 2013). HSCs are thereby ultimately converted into proliferative, fibrogenic myofibroblasts that express $\alpha$-SMA (Hinz et al., 2007; Friedman, 2008). HSC-derived myofibroblasts upregulate matrix deposition, including fibrillary types I, III, and IV collagen that are characteristic of all fibrotic diseases and are each increased up to 10 -fold in advanced liver fibrosis (Westergren-Thorsson et al., 1993; Lucey et al., 1998; Schuppan et al., 2001; Gressner and Weiskirchen, 2006; Hinz et al., 2007; Kisseleva and Brenner, 2008). Upon liver damage, HSCderived myofibroblasts proliferate and migrate to the site of 


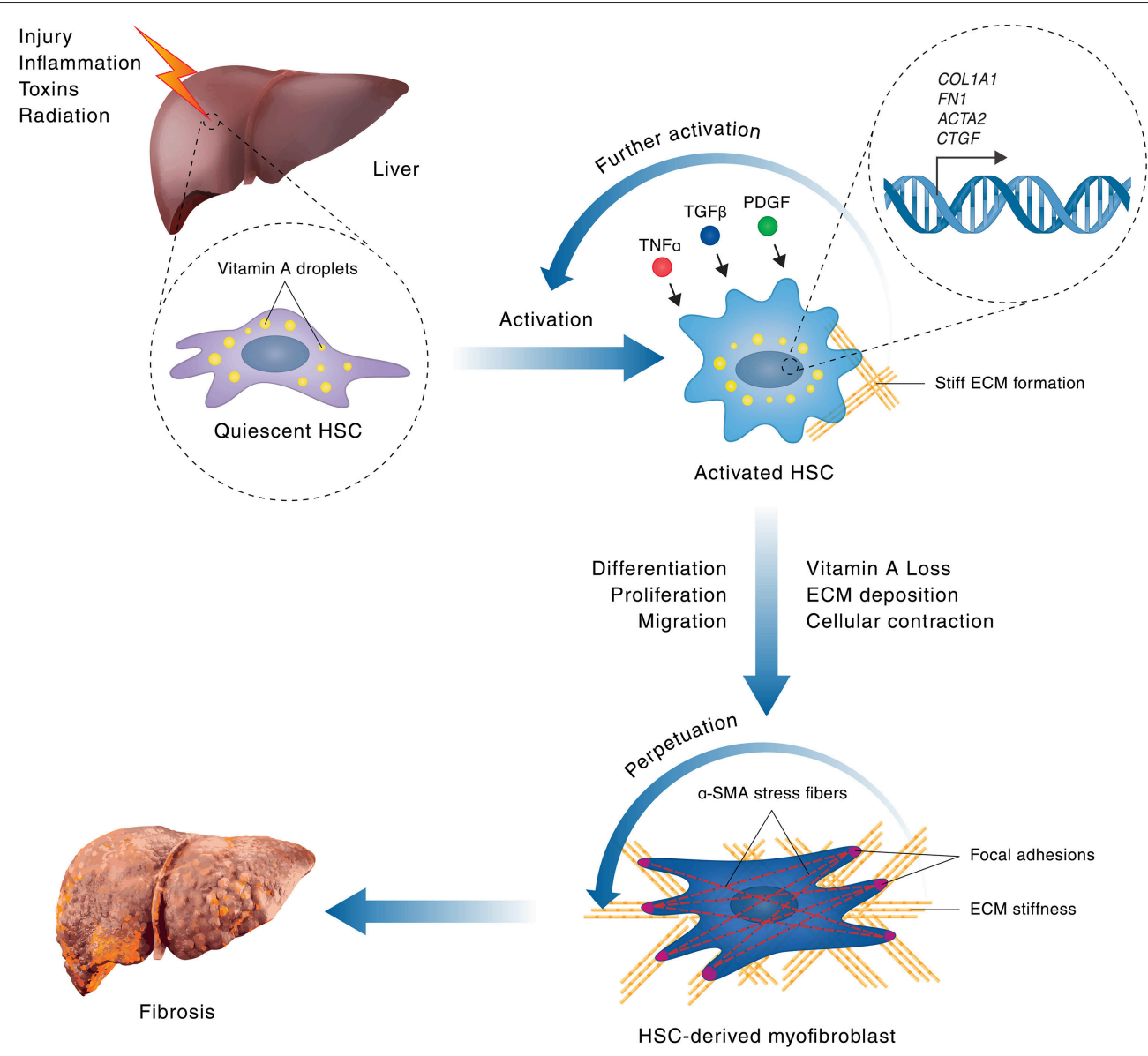

FIGURE 1 | Hepatic stellate cell activation in liver fibrosis. A schematic model of HSC activation in fibrosis is shown. Hepatocellular injuries or inflammation lead to activation of quiescent, vitamin A-rich, HSCs via signaling processes that involve cytokines, such as TNF- $\alpha$, TGF- $\beta 1$ and PDGF. HSC activation leads to upregulation of gene expression of genes encoding pro-proliferative, cytoskeletal and extracellular matrix components, such as Acta2 (encoding aSMA), Col1A1, FN1 (encoding Fibronectin 1), and Ctgf, leading to subsequent stimulation of HSC proliferation and extracellular matrix secretion. During chronic liver injury, a positive feedback loop of self-sustained, pathological HSC activation is stimulated where increased HSC proliferation leads to excessive deposition of extracellular matrix. The resulting increase in matrix stiffness promotes further stress fiber and focal adhesion formation in HSCs by mechanotransduction, leading to increased contractility, thus perpetuating HSC activation and promoting their transformation into proliferative, fibrogenic myofibroblasts, which are the main mediators of fibrosis. The persistent population of myofibroblasts, and the resultant crosslinked collagen matrix they produce, causes progressive formation of fibrous scar tissue, ultimately leading to the destruction of normal tissue architecture and the development of liver fibrosis.

injury to promote wound healing by depositing and remodeling extracellular matrix components that encapsulate the injury (Figure 1). During chronic liver injury, however, aberrant wound healing leads to hyperproliferation and reduced apoptosis of myofibroblasts. Increased contact between myofibroblasts and the extracellular matrix is mediated by $\alpha$-SMA expressioninduced stress fiber and focal adhesion (FA) formation. This increased contact leads to increased contractility, inducing mechanotransduction-related signaling (Butcher et al., 2009) and resulting in excessive extracellular matrix component deposition, cross-linking of fibrous scar tissue, and increased tissue stiffness characteristic of human fibroproliferative disease (Figure 1). Through autocrine and paracrine mechanisms, extracellular matrix components such as MMPs, fibrillary collagens and proteoglycans promote growth factor (mainly PDGF) and cytokine signaling. This signaling, particularly through the transforming growth factor-beta (TGF- $\beta$ ) and Wnt pathways, further activates HSCs, thus creating a positive, profibrotic feedback loop (Figure 1, Karsdal et al., 2015; Li et al., 2015). Ultimately, mechanotransduction- and growth factor-induced gene regulation by transcriptional regulators such as YAP and BRD4 mediate fibroproliferative disease and represents a unique target for pharmacological intervention for patients with liver fibrosis.

\section{YAP-INDUCED \\ MECHANOTRANSDUCTION PROMOTES LIVER FIBROSIS}

YAP and the homologous transcriptional co-activator with PDZbinding motif (TAZ) are the ultimate effectors of the Hippo 
pathway, a key growth control and tumor suppressor pathway originally identified by genetic studies in Drosophila melanogaster (for review see Pan, 2010; Halder and Johnson, 2011; Yu et al., 2015). The Hippo pathway is a kinase cascade in which the mammalian homologs of Hippo, the Mammalian Sterile 20-like kinases 1 and 2 (Mst1/2) activate the large tumor suppressor kinases 1 and 2 (LATS1/2), which in turn phosphorylate YAP/TAZ, thereby inducing their cytoplasmic sequestration by 14-3-3 binding and/or leading to ubiquitin-dependent degradation (Figure 2). In the absence of Hippo pathway

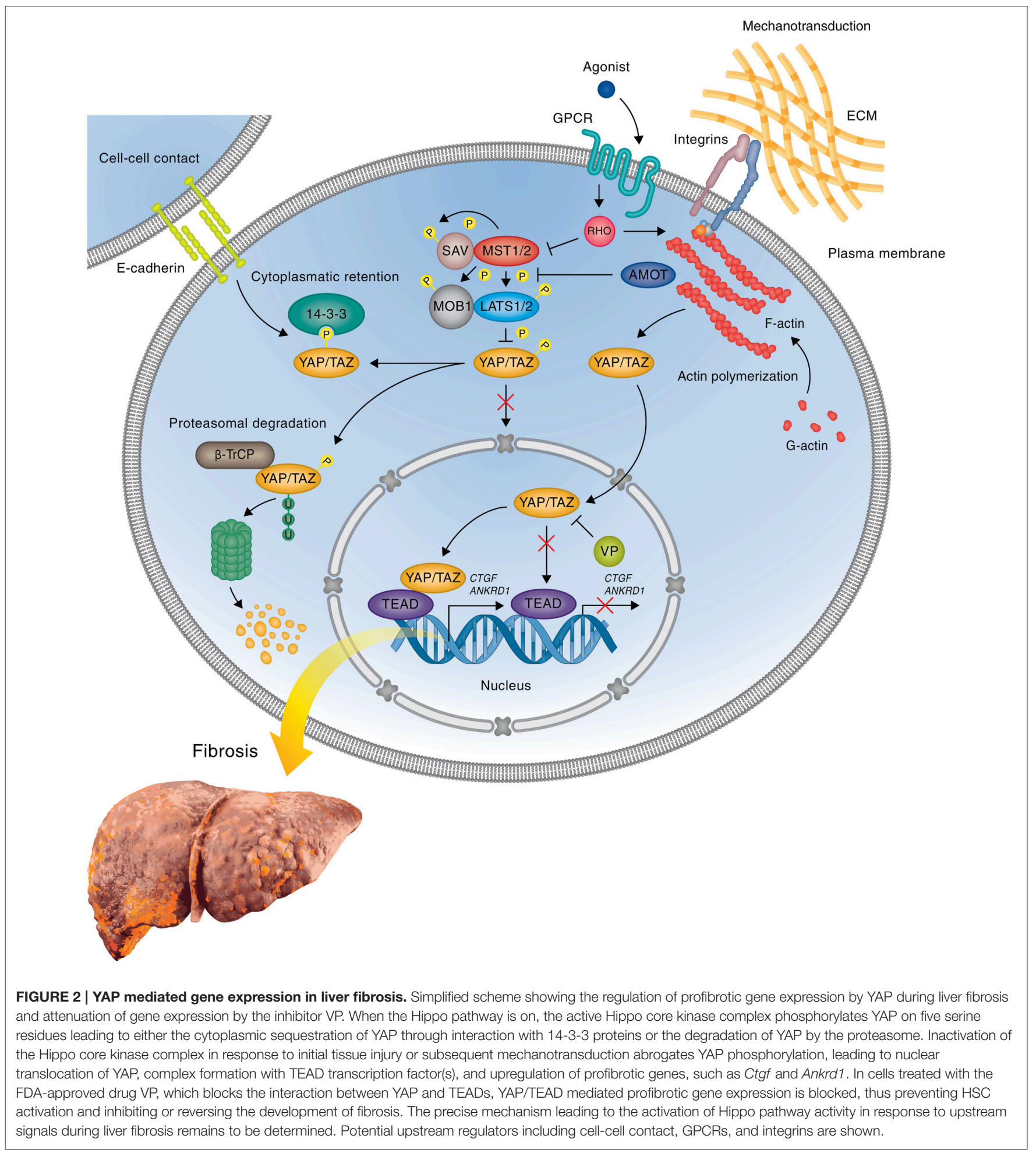


signaling activity, YAP and TAZ translocate to the nucleus where they serve as transcriptional co-activators that binds predominantly to TEAD family transcription factors (TEAD14) and promote target gene expression (Yu et al., 2015; Pan, 2010; Halder and Johnson, 2011). YAP/TAZ/TEAD regulated target genes encode proteins involved in cell proliferation and inhibition of apoptosis (for review see Johnson and Halder, 2014). Thereby, Hippo signaling regulates the balance between cell proliferation, apoptosis, and differentiation to control stem cell proliferation, organ size, as well as tissue homeostasis (Johnson and Halder, 2014). Pathologically, loss of Hippo signaling activity and hyperactivation of its downstream effectors YAP and TAZ through mutations or epigenetic regulation lead to tissue overgrowth and cancer development (Johnson and Halder, 2014).

The Hippo pathway is primarily regulated by mechanical inputs from the microenvironment (Figure 2), including cellcell contact, matrix stiffness and mechanical stress (stretch or contraction) (for review see Dupont et al., 2011; Meng et al., 2016). In addition, Hippo pathway activity is modulated both, positively and negatively, by G-protein coupled receptors (GPCRs), select cytokine receptors, such as leukemia inhibitory factor receptor, and crosstalk with specific signaling pathways, such as the EGF, Wnt, and bone morphogenic protein (BMP) pathways (Figure 2, Pan, 2010; Halder and Johnson, 2011; Yu et al., 2015; Meng et al., 2016). Mechanical cues are transmitted through activation of cell surface receptors, such as E-cadherins, integrins, and components of the cell polarity complex, as well as certain GPCRs that act as mechanoreceptors (Dupont et al., 2011; Meng et al., 2016). These receptors converge on RhoA and RhoA-dependent regulation of F-actin polymerization and actin-myosin contractility (Dupont et al., 2011; Meng et al., 2016). Cytoskeletal reorganization and contraction, in turn, alter the activity of the cytoplasmic core components of the Hippo pathway leading to phosphorylation-induced cytoplasmic retention and degradation of YAP/TAZ (Dupont et al., 2011; Meng et al., 2016). F-actin architecture is also directly linked to YAP regulation via the Hippo pathway component angiomotin (AMOT) and the related AMOTL1 and AMOTL2 (Chan et al., 2011; Zhao et al., 2011). AMOT family proteins act as adaptors that recruit YAP/TAZ to various cellular compartments, such as cell-cell junctions and Factin fibers, depending on cellular context (Chan et al., 2011; Dupont et al., 2011; Zhao et al., 2011). Disruption of junction complexes or depolymerization of F-actin releases YAP/TAZ from sequestration by AMOT and drives YAP/TAZ to the nucleus (Chan et al., 2011; Dupont et al., 2011; Zhao et al., 2011). Thus, Hippo pathway activity transforms mechanical signals from the environment through cytoskeletal reorganization into changes in nuclear expression by controlling YAP/TAZ stability and nucleocytoplasmic shuttling. Accordingly, extracellular matrix stiffness affects YAP/TAZ activity and localization, with active YAP/TAZ localizing predominantly in the nucleus in cells grown on a stiff surface, whereas YAP/TAZ remain inactive in the cytoplasm in cells grown on a soft surface (Dupont et al., 2011).

In the mammalian liver, Hippo pathway signaling is involved in development and regeneration as well as pathological processes, such as cancer and fibrosis. During liver development, YAP is important for bile duct formation (tubulogenesis), although the molecular mechanism remains to be elucidated; no effect has been observed from YAP deletion in the adult liver to date (Zhang et al., 2010; Nguyen et al., 2015). However, YAP plays a positive role in the regeneration response following bile duct ligation. Accordingly, nuclear localization of YAP increases after bile duct ligation in mice, and knockout of YAP delays hepatocyte proliferation 5 days post-injury and inhibits biliary epithelial cell proliferation 15 days post-injury (Bai et al., 2012). Conversely, YAP hyperactivation through epigenetic regulation and amplification is common in liver cancer and a wide variety of other cancers, thereby promoting tumorigenesis through cell proliferation, survival and maintenance of the tumor stem cell phenotype (Johnson and Halder, 2014).

YAP is expressed in the hepatocytes of cholestatic livers in young patients (Anakk et al., 2013) as well as in patients with primary sclerosing cholangitis and primary biliary cirrhosis (Bai et al., 2012). Liver diseases often affect the levels of serum bile acids and a recent study reported a direct association of elevated bile acid levels with YAP activation and proliferation of liver progenitor cells, eventually leading to the development of spontaneous liver tumors (Anakk et al., 2013). Importantly, YAP activation is also an early event in HSC activation and fibrosis development. An elegant study by Mannaerts et al., recently demonstrated that YAP drives HSC activation during hepatocellular injury $\left(\mathrm{CCl}_{4}\right)$ induced fibrosis in mice (Mannaerts et al., 2015). The authors showed that YAP translocates into the nuclei of $\mathrm{HSCs}$ after $\mathrm{CCl}_{4}$ administration, and inhibition of YAP through knockdown or pharmacological inhibition with verteporfin (VP) prevents HSC activation in vitro and fibrogenesis in vivo (Mannaerts et al., 2015). Knockdown studies and pharmacological inhibition of upstream Hippo pathway components further show that YAP stability and nuclear localization during HSC activation is regulated through modulation of Hippo pathway activity (Figure 2, Mannaerts et al., 2015). The activation of HSC correlates with a switch in gene expression that involves upregulation of genes, which participate in matrix remodeling, actin cytoskeleton, cell proliferation, and immune processes. Importantly, some of the most strongly upregulated genes, such as Ankrd1 (cardiac ankyrin repeat protein) and Ctgf (connective tissue growth factor), are direct targets of YAP (Figure 2, Mannaerts et al., 2015). Ankrd1 is a mechanosensitive transcription factor that is the most highly expressed in the heart and muscle and mediates TGF- $\beta$ signaling in response to injury and stress (Kojic et al., 2011), whereas CTGF is a cysteine-rich extracellular matrix protein that plays a central role in tissue remodeling and wound repair by regulating the induction of genes, such as fibronectin, collagens (types I, III, IV, and VI) and binding to various cell surface receptors, including $\alpha \mathrm{V} \beta 3$ and $\alpha 5 \beta 1$ integrins (Lau, 2016). Notably, CTGF promotes HSC activation and proliferation and plays a critical role in fibrotic disease (Gressner and Gressner, 2008; Jun and Lau, 2011; Huang and Brigstock, 2012). Significantly, Ankrd1 and Ctgf were upregulated much earlier than Acta2 (Mannaerts et al., 2015), a widely used marker of HSC activation, consistent with the idea 
that YAP controls a very early stage in HSC activation. Similar to $\mathrm{CCl}_{4}$ treatment, YAP protein and mRNA expression is increased in the livers of mice 5 days after bile duct ligation, and inhibition of YAP through liver-specific conditional deletion reduced bile duct and hepatocyte proliferation, and enhanced hepatocyte necrosis, likely through a Survivin-dependent mechanism (Bai et al., 2012). Thus, YAP regulates the early stages of both, acute, injury-induced, and chronic, cholestatic HSC activation.

During the fibrotic response, YAP serves as a mechanotransduction mediator, integrating signaling responses to mechanical cues to promote proliferation, survival, and extracellular matrix deposition (Dupont et al., 2011; Mannaerts et al., 2015). HSC activation-mediated extracellular matrix stiffening leads to YAP activation, which, in turn, leads to additional extracellular matrix deposition and matrix stiffness ultimately resulting in a feedback loop promoting sustained HSC activation and proliferation (Figure 2, Mannaerts et al., 2015). This was shown through correlative studies in mice and, more directly, using a novel 3D in spheroids system that accurately mimics HSCs activation in vitro (Mannaerts et al., 2015). Activation of HSCs by $\mathrm{CCl}_{4}$ administration to mice induced nuclear translocation of YAP, increased total YAP protein, and increased picrosirius red (collagen), $\alpha$-SMA, and ankyrin repeat domain 1 (ANKRD1) staining; this HSC activation was reversed by pharmacologic inhibition of YAP (Mannaerts et al., 2015). Importantly, this process is entirely dependent on matrix stiffness: in 3D cultured, quiescent HSCs with a soft matrix, YAP does not translocate to the nucleus after $\mathrm{CCl}_{4}$ administration, but does translocate into the nucleus after $\mathrm{CCl}_{4}$ administration to HSCs spread on tissue culture plastic - that is, a stiff matrix-leading to HSC activation as measured by Acta2, Ankrd1, and Ctgf expression (Butcher et al., 2009; Lu et al., 2012; Mannaerts et al., 2015). Overall, these studies suggest that YAP activates initial steps of HSC activation, thus inhibition of YAP could be a new way for the prevention of liver fibrosis or reverse its progression.

The role of YAP in mechanotransduction during the fibrotic response extends beyond hepatic tissues. Indeed, YAP is expressed in fibrotic, but not healthy, lung tissue (Liu et al., 2015). As in HSCs, when pulmonary fibroblasts are grown on a stiff matrix in vitro, YAP accumulates in the nucleus; likewise, knockdown of YAP impedes matrix deposition, contraction and proliferation independent of TGF$\beta$ signaling. YAP induces plasminogen activator inhibitor-1 (PAI1), promoting cell-matrix adhesion and continued nuclear accumulation of YAP, thus resulting in a positive feedback loop by which YAP mechanotransduction leads to chronic fibrogenesis (Liu et al., 2015). In contrast to the lung, YAP promotes TGF- $\beta$ signaling in fibrotic kidneys and, similar to its role in human embryonic stem cells, regulates TGF- $\beta$ signaling by retaining active SMAD2/3 in the nucleus (Varelas et al., 2008, 2010; Beyer et al., 2013). Similarly to both the liver and the lung, however, YAP promotes renal fibrogenesis by serving as a mechanotransducer. YAP-regulated TGF- $\beta$-induced profibrotic SMAD2/3 signaling is inhibited by a soft matrix, but enhanced by a stiff matrix: pharmacologic inhibition of YAP with VP inhibited TGF- $\beta$-induced SMAD2/3 signaling in vitro in rat kidney fibroblasts as well as the progression of renal fibrosis in vivo in a murine unilateral ureteral obstruction model (Szeto et al., 2016). In this study, VP reduced the absolute mRNA levels of YAP and its cofactor transcriptional coactivator with PDZ-binding motif (TAZ) in vitro, which is notable because VP is thought to inhibit YAP through direct binding, thereby inducing a conformational change that inhibits interactions with its essential cofactors, TEAD1-4 (Liu-Chittenden et al., 2012). Collectively, these studies strongly implicate Hippo signaling, through YAP, as a critical regulator of chronic fibroproliferative diseases through its mechanotransductive abilities.

\section{TRANSCRIPTIONAL REGULATION BY BRD4 PROMOTES LIVER FIBROSIS}

Another molecule that plays a key role in regulating profibrotic mechanotransduction at the transcriptional level is the bromodomain and extra terminal domain (BET) family member BRD4. BRD4 is a double bromodomain-containing transcriptional enhancer that binds to acetylated chromatin during interphase and mitosis, thereby serving as a co-activator for transcription of MYC, E2F, and nuclear factor- $\mathrm{B}(\mathrm{NF}-\kappa \mathrm{B})$ regulated cytokines to regulate the cell cycle by promoting $\mathrm{G} 2$ to M phase transition (reviewed in Shi and Vakoc, 2014; Wang and Filippakopoulos, 2015).

During the fibrotic response, BRD4 promotes the expression of target genes that are involved in matrix remodeling and proliferation (Figure 3). Of particular note, BRD4 promotes the expression of CollA1, which encodes Type I collagen, in response to exogenous TGF- $\beta$, thereby leading to both lung and liver fibrosis (Friedman, 2008; Mederacke et al., 2013; Tang et al., 2013; Ding et al., 2015). Other prominently induced genes encode components of the cytoskeletal apparatus including Actg2 and Acta2, which encode smooth muscle gamma-actin and alpha-actin, respectively (Ding et al., 2015). Inhibition of BRD4 by both siRNA and JQ1 (or the structurally distinct, but functionally similar, chemical BRD inhibitors I-BET-151 and PFI-1) markedly reduced profibrotic mRNA expression, particularly that of Col1A1 and Acta2 (Figure 3), inhibited PDGF-mediated HSC proliferation, and blocked HSC activation during $\mathrm{CCl}_{4}$-induced liver fibrosis in mice (Ding et al., 2015). Similarly, pharmacologic inhibition of BRD4 with JQ1 reverses bleomycin-induced lung fibrosis in mice, including reduced tissue hydroxyproline and Collagen I staining, indicating that BRD4 inhibition can reduce tissue stiffness-associated profibrotic signaling (Tang et al., 2013).

Collagen is known to activate profibrotic responses in a matrix stiffness-dependent manner, including the formation of stress fibers by increasing Acta2 ( $\alpha$-SMA) expression and promoting profibrotic TGF- $\beta$ signaling by applying force to collagen-binding integrin receptors leading to downstream mechanotransduction and crosstalk with SMAD proteins (Kanta, 2015). Furthermore, inhibiting $\alpha v \beta 1$ integrin attenuates bleomycin-induced lung and $\mathrm{CCl}_{4}$-induced liver fibrosis in mice by inhibiting the processing of latent to active TGF- $\beta$ (Reed et al., 2015). Thus, collagen deposition leading to 


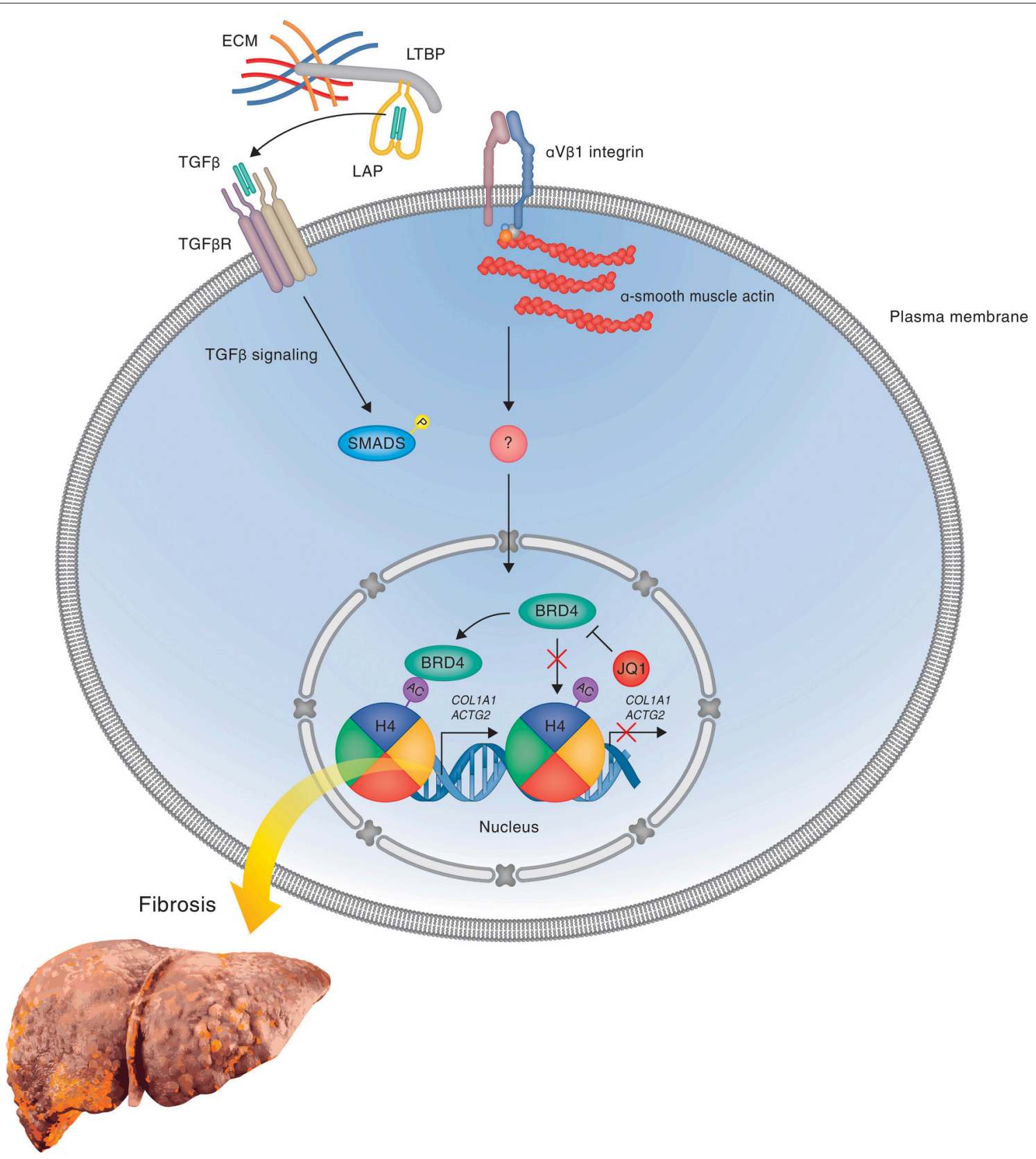

FIGURE 3 | BRD4 mediated gene expression in liver fibrosis. A hypothetical model is shown illustrating the regulation of profibrotic gene expression by the double bromodomain-containing transcriptional enhancer BRD4 during liver fibrosis in response to mechanotransduction and attenuation of gene expression by the bromodomain inhibitor JQ1 that prevents the binding of BRD4 to acetylated chromatin. During the fibrotic response, BRD4 binds to acetylated chromatin via its bromodomains and thereby promotes the expression of genes, including Col1A1, that are involved in HSC activation, proliferation and matrix remodeling. In the presence of competitive BRD inhibitors, such as JQ1, I-BET (not shown), and PFI-1 (not shown), BRD4 is prevented from binding to acetylated histones, leading to a block in transcription elongation and inhibition of TGF- $\beta$-stimulated profibrotic gene expression, thereby attenuating HSC activation and fibrosis development. The precise mechanisms leading to activation of BRD4-mediated transcriptional elongation in response to activation of profibrotic signaling pathways remains to be determined. One potential mechanism involving latent TGF- $\beta$ activation by integrins is shown.

integrin activation and mechanotransduction represents a feedback loop promoting pathologic extracellular matrix deposition and sustained profibrotic responses. Considering that BRD4 regulates TGF- $\beta$-mediated collagen deposition during the fibrotic response, an attractive mechanistic hypothesis is that BRD4 exacerbates TGF- $\beta$-mediated fibrosis and tissue stiffness by increasing signaling through integrin-mediated mechanotransductive pathways, 
leading to pathologic extracellular matrix remodeling, enhanced tissue stiffness and sustained HSC activation (Figure 3).

\section{TARGETING MATRIX STIFFNESS-INDUCED GENE EXPRESSION TO TREAT LIVER FIBROSIS}

While YAP and BRD4 appear to regulate the expression of distinct target genes and therefore likely represent two independent pathways with unique molecular mechanisms, each is regulated by the microenvironment, and each promotes extracellular matrix stiffness and mechanotransductive signaling during liver fibrosis by serving as a coactivator for profibrotic gene transcription. Likewise, each represents a novel target in the treatment of liver fibrosis that can be inhibited by targeting protein-protein interactions with co-activators via small molecules. YAP transcriptional activity can be inhibited with VP. Originally developed as a photosensitizer that is used in FDA-approved photocoagulation therapy for macular degeneration, VP has been found to bind to YAP and inhibit its interactions with TEAD proteins, which are necessary for its effects on transcriptional activation (LiuChittenden et al., 2012). VP has been demonstrated to block YAP-induced liver tumorigenesis and suppress hepatomegaly caused by hepatocyte and biliary cell proliferation in YAP transgenic and NF2 knockout mice (Liu-Chittenden et al., 2012; Nguyen et al., 2015). Furthermore, VP blocks matrix stiffness-induced HSC activation in vitro as well as $\mathrm{CCl}_{4}$ induced fibrogenesis in vivo, including a reduction in CollA1 gene expression (Mannaerts et al., 2015). As YAP is known to be expressed in human fibrotic liver and lung tissues (Liu et al., 2015; Mannaerts et al., 2015), whether pharmacologic inhibition of YAP by VP can provide a therapeutic effect for patients with fibrosis is an attractive area for further study.

Similarly to the mechanism by which VP inhibits YAP, the competitive bromodomain inhibitor JQ1 inhibits binding between Histone $\mathrm{H} 4$ and bomodomains, thereby preventing BRD4 from acting as a transcriptional co-activator of target genes (reviewed in Filippakopoulos and Knapp, 2014). JQ1 was originally developed as an antitumor drug via a small molecule screen, and has proven effective in treating in vivo models of acute myeloid leukemia, multiple myeloma and nuclear protein of the testis (NUT) midline carcinomas (reviewed in Filippakopoulos and Knapp, 2014; Wang and Filippakopoulos, 2015). In the context of fibrosis, inhibiting BRD4 with JQ1 inhibits both lung and liver fibrosis as well as HSC activation in murine models, including reducing TGF- $\beta$-mediated CollA1 expression and extracellular matrix remodeling (Tang et al., 2013; Ding et al., 2015). Thus, JQ1 represents an attractive small molecule for further study as potential treatment of human fibroproliferative diseases and the future development of more specific and stable BRD4 inhibitors (Theodoulou et al., 2016; Waring et al., 2016) may open new opportunities for anti-fibrotic therapy.

\section{CONCLUSIONS AND FUTURE PROSPECTS}

The activation of quiescent HSCs and their transition to a proliferative, contractile myofibroblast phenotype represents a critical control point between a normal and pathological tissue repair response. Few regulatory factors have been identified that regulate this transition in the liver and that can be targeted for liver fibrosis reversal. Recent progress has shown that mechanotransduction induced by increased contact between activated hepatic myofibroblasts due to pathologic accumulation of extracellular matrix components and increased focal adhesion formation is orchestrated, in part, by the transcriptional co-activators YAP and BRD4 in concert with their respective transcription factors. Traditionally, small molecule inhibitor development has focused on disrupting ligand-receptor interactions rather than transcriptional regulation. However, no therapies have been developed to date that can reverse liver fibrosis in patients, which has become a major clinical challenge in the industrialized world. More effective therapies are therefore critically needed to treat chronic fibroproliferative diseases. Given that VP and JQ1 have shown efficacy in in vivo models of liver fibrosis, targeting the fibrotic response at the transcriptional level presents an attractive avenue for further research.

BRD4 and YAP both regulate profibrotic gene expression that plays a central role in HSC activation, tissue remodeling and liver fibrosis. Nevertheless, these nuclear co-factors appear to regulate distinct sets of target genes suggesting that BRD4 and YAP represent independent pathways that are both critically involved in the regulation of early stages of fibrosis. Thus, there appear to be multiple positive feedback loops involving integrins, TGF- $\beta$, and other cytokines that contribute to HSC activation and fibrosis progression by regulating specific nuclear gene expression programs through YAP and BRD4. The studies by Mannaerts et al. (2015) and Ding et al. (2015) suggest that by inhibiting either YAP or BRD4 action, these positive feedback loops can be broken, leading to attenuation or reversal of fibrosis. If true, the rational combinations of YAP and BRD4 inhibitors may provide synergistic effects and offer a more effective way to reverse fibrosis progression than the single agents and this should be a further avenue of research. Toward this aim, it will be important to confirm the roles of YAP and BRD4 in fibrosis development and reversal using inducible knockout mouse mutants for YAP and BRD4 and to evaluate in more detail the anti-fibrotic action and efficacy of VP and JQ1 in disease relevant animal models. In addition, a more detailed understanding of the regulation of and interaction between YAP and $\mathrm{BRD} 4$ pathways will be necessary to define the precise roles of these pathways in HSC activation and fibrosis. While YAP and BRD4 each appear to control different populations of target genes, it is likely that the two pathways cooperate during HSC activation. Indeed, a recent study using the BRD inhibitor JQ1 revealed a role of BET bromodomain protein(s) in regulating the activity of the YAP homolog TAZ (Duan et al., 2016) indicating a possible point of cross-talk between the two profibrotic pathways. 
Last, but not least, of particular interest will be the elucidation of the precise mechanisms by which mechanical signals from the environment are relayed across the membrane to modulate YAP and BRD4 function in the nucleus. Integrins are the primary mechanosensors and transducers of tissue stiffness and several integrins have been identified as key drivers of profibrotic signaling in various organs. Some of these integrins, in particular $\alpha \mathrm{v}$ subunit integrins, such as $\alpha \mathrm{v} \beta 1$ and $\alpha \mathrm{v} \beta 6$, have been shown to control the activation of latent TGF- $\beta 1$ complex and targeting these integrins with small molecule inhibitors can block or reverse fibrosis progression in mouse models (Henderson and Sheppard, 2013; Henderson et al., 2013; Reed et al., 2015; Conroy et al., 2016). Given that BRD4 is critical for TGF- $\beta$-dependent profibrotic gene expression, BRD4 activity may be one of the downstream targets of integrin and TGF- $\beta$ action.

Alternatively, integrins can control HSC activation through bi-directional "outside-in" and "inside-out" signaling mechanisms leading to changes in actin organization and actomyosin contractility and subsequent alterations in tissue remodeling. Indeed, several integrin-associated signaling proteins, including integrin-linked kinase (ILK, Zhang et al., 2006; Li et al., 2009; Kavvadas et al., 2010; Shafiei and Rockey, 2012; Radovanac et al., 2013), focal adhesion kinase (FAK, Wong et al., 2011; Lagares et al., 2012; Kinoshita et al., 2013; Lagares and Kapoor, 2013; Zhao et al., 2016), and regulators and effectors of Rho GTPases, such as P-Rex1 (Liang et al., 2016), PAK and Rho-activated kinase (ROCK, Knipe et al., 2015; Martin et al., 2016) have been identified as potential targets for anti-fibrotic therapy in a number of organs. Notably, activation of ILK, which links F-actin to focal adhesions and is required for integrinmediated force generation, sustains nuclear YAP accumulation in pulmonary arterial vascular smooth muscle cells to promote cell proliferation in pulmonary arterial hypertension, a disease characterized by increased deposition of extracellular matrix and vascular stiffness (Kudryashova et al., 2016). Consistent with this notion, regulation of Hippo activation by ILK (Serrano et al., 2013), Src-FAK (Kim and Gumbiner, 2015; Li et al., 2016), and betal integrins (Martin et al., 2016) has been observed in the context of epithelial-mesenchyme transition, cancer, as well as tissue homeostasis in the skin (Elbediwy et al., 2016). Although the physiological relevance of Hippo pathway regulation by

\section{REFERENCES}

Anakk, S., Bhosale, M., Schmidt, V. A., Johnson, R. L., Finegold, M. J., and Moore, D. D. (2013). Bile acids activate YAP to promote liver carcinogenesis. Cell Rep. 5, 1060-1069. doi: 10.1016/j.celrep.2013.10.030

Bai, H., Zhang, N., Xu, Y., Chen, Q., Khan, M., Potter, J. J., et al. (2012). Yes-associated protein regulates the hepatic response after bile duct ligation. Hepatology 56, 1097-1107. doi: 10.1002/hep.25769

Ballestri, S., Nascimbeni, F., Romagnoli, D., Baldelli, E., Targher, G., and Lonardo, A. (2016). Type 2 diabetes in non-alcoholic fatty liver disease and hepatitis C virus infection-liver: the "Musketeer" in the spotlight. Int. J. Mol. Sci. 17:355. doi: $10.3390 /$ ijms 17030355

Beyer, T. A., Weiss, A., Khomchuk, Y., Huang, K., Ogunjimi, A. A., Varelas, X., et al. (2013). Switch enhancers interpret TGF- $\beta$ and Hippo signaling to control cell fate in human embryonic stem cells. Cell Rep. 5, 1611-1624. doi: 10.1016/j. celrep.2013.11.021 integrins and their effectors needs to be confirmed in the context of fibrosis there is accumulating evidence supporting such a role. Indeed, while this review was under consideration, the integrin $\alpha 11 \beta 1$ was reported to promote liver fibrosis by modulating hepatic stellate cell activation through the Hippo/YAP pathway (Martin et al., 2016). Interestingly, knockdown of the $\alpha 11$ subunit in HSCs by RNA interference abolished a subset of YAPregulated genes during HSC activation and partially reversed tissue fibrosis in a mouse model of the disease, indicating that this particular integrin represents one of likely multiple mechanosensitive upstream regulators of YAP in liver fibrosis (Martin et al., 2016).

In sum, mechanotransduction in myofibroblasts is clearly relevant for fibrotic disease and it is increasingly being realized that components of mechanosensitive signal transduction pathways are not only drivers of disease progression, but also promising targets for therapy. While additional work will be required to address the precise roles of YAP and BRD4 in fibrosis, overall the current findings suggest that YAP and BRD4 are key terminal effectors of mechanosensing signal transduction pathways that initiate and perpetuate fibrosis and that targeting YAP and BRD4 alone or in combination has great promise toward treating patients with organ fibrosis and reducing the burden of fibroproliferative diseases. A more refined understanding of the mechanisms underlying myofibroblast activation across different organ systems and better insight into the roles and regulation of YAP and BRD4 in this process could lead to more personalized treatment options that may slow and perhaps even reverse fibrosis progression in the future.

\section{AUTHOR CONTRIBUTIONS}

AZ, AT, KK, LW, and JK have written this review. All authors have read the review and gave their agreement for submission.

\section{ACKNOWLEDGMENTS}

We thank Georg Halder (VIB; Leuven) for comments on the manuscript. This work was supported by a Social Policy Fund grant and an ORAU award from Nazarbayev University (both awarded to JK).

Butcher, D. T., Alliston, T., and Weaver, V. M. (2009). A tense situation: forcing tumour progression. Nat. Rev. Cancer 9, 108-122. doi: 10.1038/ $\operatorname{nrc} 2544$

Chan, S. W., Lim, C. J., Chong, Y. F., Pobbati, A. V., Huang, C., and Hong, W. (2011). Hippo pathway-independent restriction of TAZ and YAP by angiomotin. J. Biol. Chem. 286, 7018-7026. doi: 10.1074/jbc.C110. 212621

Conroy, K. P., Kitto, L. J., and Henderson, N. C. (2016). av integrins: key regulators of tissue fibrosis. Cell Tissue Res. 365, 511-519. doi: 10.1007/s00441-0162407-9

Ding, N., Hah, N., Yu, R. T., Sherman, M. H., Benner, C., Leblanc, M., et al. (2015). BRD4 is a novel therapeutic target for liver fibrosis. Proc. Natl. Acad. Sci. U.S.A. 112, 15713-15718. doi: 10.1073/pnas.1522163112

Duan, Q., Xiao, Y., Zhu, L., Liu, Z., Mao, X., Zhou, Z., et al. (2016). BET bromodomain is a novel regulator of TAZ and its activity. Biochim. Biophys. Acta. 1859, 1527-1537. doi: 10.1016/j.bbagrm.2016.10.001 
Dupont, S., Morsut, L., Aragona, M., Enzo, E., Giulitti, S., Cordenonsi, M., et al. (2011). Role of YAP/TAZ in mechanotransduction. Nature 474, 179-183. doi: $10.1038 /$ nature 10137

Elbediwy, A., Vincent-Mistiaen, Z. I., Spencer-Dene, B., Stone, R. K., Boeing, S., Wculek, S. K., Cordero, J., et al. (2016). Integrin signalling regulates YAP and TAZ to control skin homeostasis. Development 143, 1674-1687. doi: 10.1242/ dev. 133728

Filippakopoulos, P., and Knapp, S. (2014). Targeting bromodomains: epigenetic readers of lysine acetylation. Nat. Rev. Drug Discov. 13, 337-356. doi: 10.1038/ $\operatorname{nrd} 4286$

Friedman, S. L. (2008). Hepatic stellate cells: protean, multifunctional, and enigmatic cells of the liver. Physiol. Rev. 88, 125-172. doi: 10.1152/physrev. 00013.2007

Friedman, S. L., Sheppard, D., Duffield, J. S., and Violette, S. (2013). Therapy for fibrotic diseases: nearing the starting line. Sci. Transl. Med. 5, 167sr1. doi: 10. 1126/scitranslmed.3004700

Gressner, A. M., and Weiskirchen, R. (2006). Modern pathogenetic concepts of liver fibrosis suggest stellate cells and TGF- $\beta$ as major players and therapeutic targets. J. Cell. Mol. Med. 10, 76-99. doi: 10.1111/j.1582-4934.2006.tb00292.x

Gressner, O. A., and Gressner, A. M. (2008). Connective tissue growth factor: a fibrogenic master switch in fibrotic liver diseases. Liver Int. 28, 1065-1079. doi: 10.1111/j.1478-3231.2008.01826.x

Halder, G., and Johnson, R. L. (2011). Hippo signaling: growth control and beyond. Development 138, 9-22. doi: 10.1242/dev.045500

Henderson, N. C., Arnold, T. D., Katamura, Y., Giacomini, M. M., Rodriguez, J. D., McCarty, J. H., et al. (2013). Targeting of $\alpha \mathrm{v}$ integrin identifies a core molecular pathway that regulates fibrosis in several organs. Nat. Med. 19, 1617-1624. doi: $10.1038 / \mathrm{nm} .3282$

Henderson, N. C., and Sheppard, D. (2013). Integrin-mediated regulation of TGF $\beta$ in fibrosis. Biochim. Biophys. Acta 1832, 891-896. doi: 10.1016/j.bbadis.2012. 10.005

Hinz, B., Phan, S. H., Thannickal, V. J., Galli, A., Bochaton-Piallat, M. L., and Gabbiani, G. (2007). The myofibroblast: one function, multiple origins. Am. J. Pathol. 170, 1807-1816. doi: 10.2353/ajpath.2007.070112

Huang, G., and Brigstock, D. R. (2012). Regulation of hepatic stellate cells by connective tissue growth factor. Front. Biosci. (Landmark Ed) 17, 2495-2507. doi: $10.2741 / 4067$

Iwaisako, K., Jiang, C., Zhang, M., Cong, M., Moore-Morris, T. J., Park, T. J., et al. (2014). Origin of myofibroblasts in the fibrotic liver in mice. Proc. Natl. Acad. Sci. U.S.A. 111, E3297-E3305. doi: 10.1073/pnas.1400062111

Johnson, R., and Halder, G. (2014). The two faces of Hippo: targeting the Hippo Pathway for regenerative medicine and cancer treatment. Nat. Rev. Drug Discov. 13, 63-79. doi: 10.1038/nrd4161

Jun, J. I., and Lau, L. F. (2011). Taking aim at the extracellular matrix: CCN proteins as emerging therapeutic targets. Nat. Rev. Drug Discov. 10, 945-963. doi: 10. $1038 /$ nrd3599

Kanta, J. (2015). Collagen matrix as a tool in studying fibroblastic cell behavior. Cell Adh. Migr. 9, 308-316. doi: 10.1080/19336918.2015.1005469

Karsdal, M. A., Manon-Jensen, T., Genovese, F., Kristensen, J. H., Nielsen, M. J., Sand, J. M., et al. (2015). Novel insights into the function and dynamics of extracellular matrix in liver fibrosis. Am. J. Physiol. Gastrointest. Liver Physiol. 308, G807-G830. doi: 10.1152/ajpgi.00447.2014

Kavvadas, P., Kypreou, K. P., Protopapadakis, E., Prodromidi, E., Sideras, P., and Charonis, A. S. (2010). Integrin-linked kinase (ILK) in pulmonary fibrosis. Virchows Arch. 457, 563-575. doi: 10.1007/s00428-010-0976-7

Kim, N. G., and Gumbiner, B. M. (2015). Adhesion to fibronectin regulates Hippo signaling via the FAK-Src-PI3K pathway. J. Cell Biol. 210, 503-515. doi: 10. 1083/jcb.201501025

Kinoshita, K., Aono, Y., Azuma, M., Kishi, J., Takezaki, A., Kishi, M., et al. (2013). Antifibrotic effects of focal adhesion kinase inhibitor in bleomycin-induced pulmonary fibrosis in mice. Am. J. Respir. Cell Mol. Biol. 49, 536-543. doi: 10. 1165/rcmb.2012-0277OC

Kisseleva, T., and Brenner, D. A. (2008). Fibrogenesis of parenchymal organs. Proc. Am. Thorac. Soc. 5, 338-342. doi: 10.1513/pats.200711-168DR

Knipe, R. S., Tager, A. M., and Liao, J. K. (2015). The Rho kinases: critical mediators of multiple profibrotic processes and rational targets for new therapies for pulmonary fibrosis. Pharmacol. Rev. 67, 103-117. doi: 10.1124/pr.114.009381
Kojic, S., Radojkovic, D., and Faulkner, G. (2011). Muscle ankyrin repeat proteins: their role in striated muscle function in health and disease. Crit. Rev. Clin. Lab. Sci. 48, 269-294. doi: 10.3109/10408363.2011.643857

Kudryashova, T. V., Goncharov, D. A., Pena, A., Kelly, N., Vanderpool, R., Baust, J., et al. (2016). Hippo-integrin-linked kinase cross-talk controls self-sustaining proliferation and survival in pulmonary hypertension. Am. J. Respir. Crit. Care Med. 194, 866-877. doi: 10.1164/rccm.201510-2003OC

Lagares, D., Busnadiego, O., Garcia-Fernandez, R. A., Kapoor, M., Liu, S., Carter, D. E., et al. (2012). Inhibition of focal adhesion kinase prevents experimental lung fibrosis and myofibroblast formation. Arthritis Rheum. 64, 1653-1664. doi: 10.1002/art.33482

Lagares, D., and Kapoor, M. (2013). Targeting focal adhesion kinase in fibrotic diseases. BioDrugs 27, 15-23. doi: 10.1007/s40259-012-0003-4

Lau, L. F. (2016). Cell surface receptors for CCN proteins. J. Cell Commun. Signal. 10, 121-127. doi: 10.1007/s12079-016-0324-Z

Li, H. Y., Ju, D., Zhang, D. W., Li, H., Kong, L. M., Guo, Y., et al. (2015). Activation of TGF- $\beta 1-C D 147$ positive feedback loop in hepatic stellate cells promotes liver fibrosis. Sci. Rep. 5:16552. doi: 10.1038/srep16552

Li, P., Silvis, M. R., Honaker, Y., Lien, W. H., Arron, S. T., and Vasioukhin, V. (2016). $\alpha$ E-catenin inhibits a Src-YAP1 oncogenic module that couples tyrosine kinases and the effector of Hippo signaling pathway. Genes Dev. 30, 798-811. doi: $10.1101 / \operatorname{gad} .274951 .115$

Li, Y., Tan, X., Dai, C., Stolz, D. B., Wang, D., and Liu, Y. (2009). Inhibition of integrin-linked kinase attenuates renal interstitial fibrosis. J. Am. Soc. Nephrol. 20, 1907-1918. doi: 10.1681/ASN.2008090930

Liang, Q., Cheng, N., Zhang, G., Liang, Y., Qian, F., Wu, D., et al. (2016). Identification of P-Rex1 as an anti-inflammatory and anti-fibrogenic target for pulmonary fibrosis. Sci. Rep. 6:25785. doi: 10.1038/srep25785

Liu, F., Lagares, D., Choi, K. M., Stopfer, L., Marinkovic, A., Vrbanac, V., et al. (2015). Mechanosignaling through YAP and TAZ drives fibroblast activation and fibrosis. Am. J. Physiol. Lung Cell. Mol. Physiol. 308, L344-L357. doi: 10. 1152/ajplung.00300.2014

Liu, X., Xu, J., Brenner, D. A., and Kisseleva, T. (2013). Reversibility of liver fibrosis and inactivation of fibrogenic myofibroblasts. Curr. Pathobiol. Rep. 1, 209-214. doi: 10.1007/s40139-013-0018-7

Liu-Chittenden, Y., Huang, B., Shim, J. S., Chen, Q., Lee, S. J., Anders, R. A., et al. (2012). Genetic and pharmacological disruption of the TEAD-YAP complex suppresses the oncogenic activity of YAP. Genes Dev. 26, 1300-1305. doi: 10. 1101/gad.192856.112

Lu, P., Weaver, V. M., and Werb, Z. (2012). The extracellular matrix: a dynamic niche in cancer progression. J. Cell Biol. 196, 395-406. doi: 10.1083/jcb. 201102147

Lucey, E. C., Goldstein, R. H., Stone, P. J., and Snider, G. L. (1998). Remodeling of alveolar walls after elastase treatment of hamsters. Results of elastin and collagen mRNA in situ hybridization. Am. J. Respir. Crit. Care Med. 158, 555-564. doi: 10.1164/ajrccm.158.2.9705021

Mannaerts, I., Leite, S. B., Verhulst, S., Claerhout, S., Eysackers, N., Thoen, L. F., et al. (2015). The Hippo Pathway effector YAP controls mouse hepatic stellate cell activation. J. Hepatol. 63, 679-688. doi: 10.1016/j.jhep.2015.04.011

Martin, K., Pritchett, J., Llewellyn, J., Mullan, A. F., Athwal, V. S., Dobie, R., et al. (2016). PAK proteins and YAP-1 signalling downstream of integrin $\beta-1$ in myofibroblasts promote liver fibrosis. Nat. Commun. 7:12502. doi: 10.1038/ ncomms 12502

Mederacke, I., Hsu, C. C., Troeger, J. S., Huebener, P., Mu, X., Dapito, D. H., et al. (2013). Fate tracing reveals hepatic stellate cells as dominant contributors to liver fibrosis independent of its aetiology. Nat. Commun. 4, 2823. doi: 10.1038/ ncomms 3823

Mehal, W. Z., Iredale, J., and Friedman, S. L. (2011). Scraping fibrosis: expressway to the core of fibrosis. Nat. Med. 17, 552-553. doi: 10.1038/nm0511-552

Meng, Z., Moroishi, T., and Guan, K. L. (2016). Mechanisms of Hippo Pathway regulation. Genes Dev. 30, 1-17. doi: 10.1101/gad.274027.115

Nguyen, Q., Anders, R. A., Alpini, G., and Bai, H. (2015). Yes-associated protein in the liver: regulation of hepatic development, repair, cell fate determination and tumorigenesis. Dig. Liver Dis. 47, 826-835. doi: 10.1016/j.dld.2015. 05.011

Pan, D. (2010). The Hippo signaling pathway in development and cancer. Dev. Cell 19, 491-505. doi: 10.1016/j.devcel.2010.09.011 
Poordad, F., Hezode, C., Trinh, R., Kowdley, K. V., Zeuzem, S., Agarwal, K., et al. (2014). ABT-450/r-ombitasvir and dasabuvir with ribavirin for hepatitis $C$ with cirrhosis. N. Engl. J. Med. 370, 1973-1982. doi: 10.1056/NEJMoa1402869

Radovanac, K., Morgner, J., Schulz, J. N., Blumbach, K., Patterson, C., Geiger, T., et al. (2013). Stabilization of integrin-linked kinase by the Hsp90-CHIP axis impacts cellular force generation, migration and the fibrotic response. EMBO J. 32, 1409-1424. doi: 10.1038/emboj.2013.90

Reed, N. I., Jo, H., Chen, C., Tsujino, K., Arnold, T. D., DeGrado, W. F., et al. (2015). The $\alpha v \beta 1$ integrin plays a critical in vivo role in tissue fibrosis. Sci. Transl. Med. 7, 288ra79. doi: 10.1126/scitranslmed.aaa5094

Rockey, D. C., Bell, P. D., and Hill, J. A. (2015). Fibrosis-a common pathway to organ injury and failure. N. Engl. J. Med. 373, 96. doi: 10.1056/NEJMra1300575

Schuppan, D., Ruehl, M., Somasundaram, R., and Hahn, E. G. (2001). Matrix as a modulator of hepatic fibrogenesis. Semin. Liver Dis. 21, 351-372. doi: 10.1055/ s-2001-17556

Serrano, I., McDonald, P. C., Lock, F., Muller, W. J., and Dedhar, S. (2013). Inactivation of the Hippo tumour suppressor pathway by integrin-linked kinase. Nat. Commun. 4:2976. doi: 10.1038/ncomms3976

Shafiei, M. S., and Rockey, D. C. (2012). The function of integrin-linked kinase in normal and activated stellate cells: implications for fibrogenesis in wound healing. Lab. Invest. 92, 305-316. doi: 10.1038/labinvest.2011.155

Shi, J., and Vakoc, C. R. (2014). The mechanisms behind the therapeutic activity of BET bromodomain inhibition. Mol. Cell 54, 728-736. doi: 10.1016/j.molcel. 2014.05.016

Szeto, S. G., Narimatsu, M., Lu, M., He, X., Sidiqi, A. M., Tolosa, M. F., et al. (2016). YAP/TAZ are mechanoregulators of TGF- $\beta$-Smad signaling and renal fibrogenesis. J. Am. Soc. Nephrol. 27, 3117-3128. doi: 10.1681/ASN.2015050499

Tang, X., Peng, R., Phillips, J. E., Deguzman, J., Ren, Y., Apparsundaram, S., et al. (2013). Assessment of Brd4 inhibition in idiopathic pulmonary fibrosis lung fibroblasts and in vivo models of lung fibrosis. Am. J. Pathol. 183, 470-479. doi: 10.1016/j.ajpath.2013.04.020

Theodoulou, N. H., Tomkinson, N. C., Prinjha, R. K., and Humphreys, P. G. (2016). Clinical progress and pharmacology of small molecule bromodomain inhibitors. Curr. Opin. Chem. Biol. 33, 58-66. doi: 10.1016/j.cbpa.2016. 05.028

Varelas, X., Sakuma, R., Samavarchi-Tehrani, P., Peerani, R., Rao, B. M., Dembowy, J., et al. (2008). TAZ controls Smad nucleocytoplasmic shuttling and regulates human embryonic stem-cell self-renewal. Nat. Cell Biol. 10, 837-848. doi: 10. 1038/ncb 1748

Varelas, X., Samavarchi-Tehrani, P., Narimatsu, M., Weiss, A., Cockburn, K., Larsen, B. G., et al. (2010). The crumbs complex couples cell density sensing to Hippo-dependent control of the TGF- $\beta$-SMAD pathway. Dev. Cell 19, 831-844. doi: 10.1016/j.devcel.2010.11.012
Wang, C. Y., and Filippakopoulos, P. (2015). Beating the odds: BETs in disease. Trends Biochem. Sci. 40, 468-479. doi: 10.1016/j.tibs.2015.06.002

Waring, M. J., Chen, H., Rabow, A. A., Walker, G., Bobby, R., Boiko, S., et al. (2016). Potent and selective bivalent inhibitors of BET bromodomains. Nat. Chem. Biol. 12, 1097-1104. doi: 10.1038/nchembio.2210

Westergren-Thorsson, G., Hernnas, J., Sarnstrand, B., Oldberg, A., Heinegard, D., and Malmstrom, A. (1993). Altered expression of small proteoglycans, collagen, and transforming growth factor- $\beta 1$ in developing bleomycin-induced pulmonary fibrosis in rats. J. Clin. Invest. 92, 632-637. doi: 10.1172/JCI116631

Wong, V. W., Rustad, K. C., Akaishi, S., Sorkin, M., Glotzbach, J. P., Januszyk, M., et al. (2011). Focal adhesion kinase links mechanical force to skin fibrosis via inflammatory signaling. Nat. Med. 18, 148-152. doi: 10.1038/nm.2574

Yu, F. X., Zhao, B., and Guan, K. L. (2015). Hippo Pathway in organ size control, tissue homeostasis, and cancer. Cell 163, 811-828. doi: 10.1016/j.cell.2015. 10.044

Zeuzem, S., Jacobson, I. M., Baykal, T., Marinho, R. T., Poordad, F., Bourliere, M., et al. (2014). Retreatment of HCV with ABT-450/r-ombitasvir and dasabuvir with ribavirin. N. Engl. J. Med. 370, 1604-1614. doi: 10.1056/NEJMoa1401561

Zhang, N., Bai, H., David, K. K., Dong, J., Zheng, Y., Cai, J., et al. (2010). The Merlin/NF2 tumor suppressor functions through the YAP oncoprotein to regulate tissue homeostasis in mammals. Dev. Cell 19, 27-38. doi: 10.1016/j. devcel.2010.06.015

Zhang, Y., Ikegami, T., Honda, A., Miyazaki, T., Bouscarel, B., Rojkind, M., et al. (2006). Involvement of integrin-linked kinase in carbon tetrachloride-induced hepatic fibrosis in rats. Hepatology 44, 612-622. doi: 10.1002/hep.21315

Zhao, B., Li, L., Lu, Q., Wang, L. H., Liu, C. Y., Lei, Q., et al. (2011). Angiomotin is a novel Hippo pathway component that inhibits YAP oncoprotein. Genes Dev. 25, 51-63. doi: 10.1101/gad.2000111

Zhao, X. K., Cheng, Y., Liang Cheng, M., Yu, L., Mu, M., Li, H., et al. (2016). Focal adhesion kinase regulates fibroblast migration via integrin $\beta-1$ and plays a central role in fibrosis. Sci. Rep. 6:19276. doi: 10.1038/srep19276

Conflict of Interest Statement: The authors declare that the research was conducted in the absence of any commercial or financial relationships that could be construed as a potential conflict of interest.

Copyright $\odot 2016$ Zhubanchaliyev, Temirbekuly, Kongrtay, Wanshura and Kunz. This is an open-access article distributed under the terms of the Creative Commons Attribution License (CC BY). The use, distribution or reproduction in other forums is permitted, provided the original author(s) or licensor are credited and that the original publication in this journal is cited, in accordance with accepted academic practice. No use, distribution or reproduction is permitted which does not comply with these terms. 